Vol. 53 (1996) [479-484]

\title{
A PROPERTY OF SERIES OF HOLOMORPHIC HOMOGENEOUS POLYNOMIALS WITH HADAMARD GAPS Jun SOO CHOA
}

Recently J. Miao proved that if $f(z)=\sum_{k=1}^{\infty} a_{k} z^{n_{k}}$ is a holomorphic function with Hadamard gaps on the open unit disc $D$ then $f \in X^{p}$ if and only if $f \in B^{p}$ if and only if $f \in B_{0}^{p}$ if and only if $\sum_{k=1}^{\infty}\left|a_{k}\right|^{p}<\infty$, where $X^{p}, B^{p}$ and $B_{0}^{p}$ denote respectively the class of holomorphic functions on $D$ which satisfy $\left|f^{\prime}(z)\right|^{p}\left(1-|z|^{2}\right)^{p-1} d x d y$ is a finite measure, a Carleson measure and a little Carleson measure on $\mathbb{D}$. In this paper we give a higher-dimensional version of Miao's result.

\section{INTRODUCTION}

Notation used in this note will be for the most part as in $[8]$. Let $\mathbb{B}=\mathbb{B}_{n}$ be the open unit ball of $C^{n}(n \geqslant 1)$ and $V$ be the Lebesgue volume measure on $\mathbb{B}$ normalised so thet $V(\mathbb{B})=1$. We write $S$ for the boundary of $\mathbb{B}$ and $\sigma$ for the normalised surface measure on $S$. We shall set $\mathbb{D}=\mathbb{B}_{1}$. For $z, w \in C^{n}$, we let $\langle z, w\rangle=\sum_{j=1}^{n} z_{j} \overline{w_{j}}$ denote the complex inner product in $C^{n}$ and $|z|=\langle z, z\rangle^{1 / 2}$. For a function $f$ holomorphic on $\mathbb{B}$, the radial derivative $\mathcal{R} f$ of $f$ is defined by

$$
\mathcal{R} f(z)=\sum_{j=1}^{n} z_{j} \frac{\partial f}{\partial z_{j}}(z) \quad(z \in \mathbb{B})
$$

Note that $\mathcal{R} f(z)=\sum_{k=0}^{\infty} k f_{k}(z)$ if $f$ has the homogeneous polynomial expansion $f(z)=$ $\sum_{k=0}^{\infty} f_{k}(z)$

We say that a positive measure $\mu$ on $\mathbb{B}$ is a Carleson measure (respecitvely a little Carleson measure) if

$$
\sup _{a \in B} \int_{B} \frac{\left(1-|a|^{2}\right)^{n}}{|1-\langle z, a\rangle|^{2 n}} d \mu(z)<\infty \quad\left(\text { respectively } \lim _{|a| /^{\prime 1}} \int_{B} \frac{\left(1-|a|^{2}\right)^{n}}{|1-\langle z, a\rangle|^{2 n}} d \mu(z)=0\right) .
$$

Received 3rd August, 1995

This research was partially supported by TGRC and BSRI: 95-1420. The author wishes to thank Professor Patrick Ahern for helpful discussions.

Copyright Clearance Centre, Inc. Serial-fee code: 0004-9729/96 \$A2.00+0.00. 
For $0<p<\infty$, a function $f$ holomorphic on $\mathbb{B}$ is said to be a member of $X^{p}$, $B^{p}$ or $B_{0}^{p}$, respectively, if $|\mathcal{R} f(z)|^{p}\left(1-|z|^{2}\right)^{p-1} d V(z)$ is a finite measure, a Carleson measure or a little Carleson measure. It is clear that $B_{0}^{p} \subset B^{p} \subset X^{p}$ for each $p>0$, and it is well-known that $X^{2}$ is the Hardy space $H^{2}$. (This follows from the Littlewood-Paley intergral inequalities [2, Lemma 3.2] and the trivial identity $\left.\|f\|_{H^{2}(B)}^{2}=\int_{S}\left\|f_{\zeta}\right\|_{H^{2}(\mathbb{D})} d \sigma(\zeta)\right)$. It is also known (see [4] or [9]) that $B^{2}=B M O A$ and $B_{0}^{2}=V M O A$.

The prototype of our work is the following result concerning Hadamard gap series on the open unit disc $\mathbb{D}$, which is due to Miao [6] and it was well-known (see, for example, [3, pp.44-45]) for the special case $p=2$.

THEOREM [MrAO]. If $f(z)=\sum_{k=1}^{\infty} a_{k} z^{n_{k}}$ is a holomorphic function on the open unit disc $\mathbb{D}$ that has Hadamard gaps, that is, $n_{k+1} / n_{k} \geqslant \lambda>1$ for all $k$, then

$$
f \in X^{p} \Longleftrightarrow f \in B^{p} \Longleftrightarrow f \in B_{0}^{p} \Longleftrightarrow \sum_{k=1}^{\infty}\left|a_{k}\right|^{p}<\infty
$$

In this paper we find a family of holomorphic functions on the open unit ball $\mathbb{B}$ that have the same phenomena as occurred in the theorem above. Our main result is stated in Section 3.

\section{Definitions and Preliminary Results.}

As usual, we write for $0<p<\infty$

$$
\|h\|_{p}=\left(\int_{S}|h(\zeta)|^{p} d \sigma\right)^{1 / p}
$$

and

$$
\|h\|_{\infty}=\sup _{\zeta \in S}|h(\zeta)|
$$

if $h$ is a holomorphic homogeneous polynomial on $C^{n}$ restricted to $S$. A holomorphic function $f$ on $\mathbb{B}$ with the homogenous expansion $f(z)=\sum_{k=1}^{\infty} a_{k} P_{n_{k}}(z)$ (here, each $P_{n_{k}}$ is a homogeneous polynomial of degree $n_{k}$ ) is said to have Hadamard gaps if $n_{k+1} / n_{k} \geqslant \lambda>1$ for all $k=1,2, \cdots$.

Now we collect some material which will be used later.

The first Lemma below, which was proved by the author [1, Proposition 1], gives a criterion for a function $f$ holomorphic on $\mathbb{B}$, with Hadamard gaps, to belong to the space $X^{p}$. 
LEMMA 1. Let $0<p<\infty$ and $f(z)=\sum_{k=1}^{\infty} a_{k} P_{n_{k}}(z)$ be a holomorphic function on $\mathbb{B}$ with Hadamard gaps. Then the following conditions are equivalent.

(i) $f \in X^{p}$,

(ii) $\sum_{k=1}^{\infty}\left|a_{k}\right|^{p}\left\|P_{n_{k}}\right\|_{p}^{p}<\infty$.

The next lemma is taken from [5].

Lemma 2. Let $\alpha>0,0<p<\infty, a_{k} \geqslant 0, I_{k}=\left\{n: 2^{k} \leqslant n<2^{k+1}, k \in \mathbb{N}\right\}$ and $t_{k}=\sum_{n \in I_{k}} a_{n}$. Then there exists a constant $K(p, \alpha)$ depending only on $p$ and $\alpha$ such that

$$
K(p, \alpha)^{-1} \sum_{k=0}^{\infty} 2^{-k \alpha} t_{k}^{p} \leqslant \int_{0}^{1}\left(\sum_{k=1}^{\infty} a_{k} x^{k}\right)^{p}(1-x)^{\alpha-1} d x \leqslant K(p, \alpha) \sum_{k=0}^{\infty} 2^{-k \alpha} t_{k}^{p} .
$$

We use Lemma 2 to obtain the following result which gives a sufficient condition for a function $f$ holomorphic on $\mathbb{B}$, with Hadamard gaps, to be a member of $B_{0}^{p}$. The proof below is a slight modification of that of [6, Theorem 2]. But we include it for the sake of completeness.

Lemma 3. Let $0<p<\infty$ and $f(z)=\sum_{k=1}^{\infty} a_{k} P_{n_{k}}(z)$ be a holomorphic function on $\mathbb{B}$ with Hadamard gaps. Then

$$
\sum_{k=1}^{\infty}\left|a_{k}\right|^{p}\left\|P_{n_{k}}\right\|_{\infty}^{p}<\infty \quad \text { implies } f \in B_{0}^{p} .
$$

Proof: By Lemma 2, we have

$$
\int_{0}^{1}\left(\sum_{k=1}^{\infty} n_{k}\left|a_{k}\right|\left\|P_{n_{k}}\right\|_{\infty} r^{n_{k}}\right)^{p}(1-r)^{p-1} d r \leqslant K(p) \sum_{k=0}^{\infty} 2^{-k p} t_{k}^{p}
$$

where $t_{k}=\sum_{n_{j} \in I_{k}} n_{j}\left|a_{j}\right|\left\|P_{n_{j}}\right\|_{\infty}<2^{k+1} \sum_{n_{j} \in I_{k}}\left|a_{j}\right|\left\|P_{n_{j}}\right\|_{\infty}$.

Let $n_{k+1} / n_{k} \geqslant \lambda>1$ for all $k$. Then the number of coefficients $a_{j}$ is at most $\left[\log _{\lambda} 2\right]+1$ when $n_{j} \in I_{k}$, for $k=1,2, \cdots$. Thus by Hölder's inequality we have

$$
\begin{aligned}
\sum_{k=0}^{\infty} 2^{-k p} t_{k}^{p} & \leqslant 2^{p} \sum_{k=0}^{\infty}\left(\sum_{n_{j} \in I_{k}}\left|a_{j}\right|\left\|P_{n_{j}}\right\|_{\infty}\right)^{p} \\
& \leqslant 2^{p}\left(\left[\log _{\lambda} 2\right]+1\right)^{p} \sum_{j=1}^{\infty}\left|a_{j}\right|^{p}\left\|P_{n_{j}}\right\|_{\infty}^{p},
\end{aligned}
$$


and so, by hypothesis, $\int_{0}^{1}\left(\sum_{k=1}^{\infty} n_{k}\left|a_{k}\right|\left\|P_{n_{k}}\right\|_{\infty} r^{n_{k}}\right)^{p}(1-r)^{p-1} d r$ is finite. Hence for any $\varepsilon>0$, there is a $\delta \in(0,1)$ such that

$$
\int_{\delta}^{1}\left(\sum_{k=1}^{\infty} n_{k}\left|a_{k}\right|\left\|P_{n_{k}}\right\|_{\infty} r^{n_{k}}\right)^{p}(1-r)^{p-1} d r<\varepsilon
$$

Then integration in polar coordinates and simple calculations give

$$
\begin{aligned}
& \int_{\mathbb{B}}|\mathcal{R} f(z)|^{p}\left(1-|z|^{2}\right)^{p-1} \frac{\left(1-|a|^{2}\right)^{n}}{|1-\langle z, a\rangle|^{2 n}} d V(z) \\
& =2 n \int_{S} \int_{0}^{1}\left|\sum_{k=1}^{\infty} n_{k} a_{k} P_{n_{k}}(\zeta) r^{n_{k}}\right|^{p}\left(1-r^{2}\right)^{p-1} \frac{\left(1-|a|^{2}\right)^{n}}{|1-\langle r \zeta, a\rangle|^{2 n}} r^{2 n-1} d r d \sigma(\zeta) \\
& \leqslant C(n, p) \int_{0}^{1}\left(\sum_{k=1}^{\infty} n_{k}\left|a_{k}\right|\left\|P_{n_{k}}\right\|_{\infty} r^{n_{k}}\right)^{p}(1-r)^{p-1}\left\{\int \frac{\left(1-|a|^{2}\right)^{n}}{|1-\langle r \zeta, a\rangle|^{2 n}} d \sigma(\zeta)\right\} d r \\
& \leqslant C(n, p) \int_{0}^{1}\left(\sum_{k=1}^{\infty} n_{k}\left|a_{k}\right|\left\|P_{n_{k}}\right\|_{\infty} r^{n_{k}}\right)^{p}(1-r)^{p-1} \frac{\left(1-|a|^{2}\right)^{n}}{\left(1-r^{2}|a|^{2}\right)^{n}} d r \\
& \leqslant C(n, p) \int_{0}^{\delta}\left(\sum_{k=1}^{\infty} n_{k}\left|a_{k}\right|\left\|P_{n_{k}}\right\|_{\infty} r^{n_{k}}\right)^{p}(1-r)^{p-1} \frac{\left(1-|a|^{2}\right)^{n}}{\left(1-r^{2}|a|^{2}\right)^{n}} d r+C(n, p) \varepsilon \\
& \leqslant C(n, p) \frac{\left(1-|a|^{2}\right)^{n}}{\left(1-\delta^{2}\right)^{n} \int_{0}^{1}\left(\sum_{k=1}^{\infty} n_{k}\left|a_{k}\right|\left\|P_{n_{k}}\right\|_{\infty} r^{n_{k}}\right)^{p}(1-r)^{p-1} d r+C(n, p) \varepsilon}
\end{aligned}
$$

where the symbol $C(n, p)$ is used to denote positive constants, not necessarily the same at each occurrence, depending only on $p$ and the dimension $n$. So

$$
\limsup _{|a| / 1} \int_{\mathbb{B}}|\mathcal{R} f(z)|^{p}\left(1-|z|^{2}\right)^{p-1} \frac{\left(1-|a|^{2}\right)^{n}}{|1-\langle z, a\rangle|^{2 n}} d V(z) \leqslant C(n, p) \varepsilon .
$$

Therefore $f \in B_{0}^{p}$, since $\varepsilon>0$ is arbitrary. This proves Lemma 2 .

The next result, which was also proved by the author [1, Proposition 5], shows that in general the converse of Lemma 3 above need not be true.

Proposition 4. Let $0<p<\infty$. Suppose $f(z)=\sum_{k=1}^{\infty} a_{k} P_{n_{k}}(z)$ is a holomorphic function on $\mathbb{B}$ with Hadamard gaps and $f(z)$ depends only on fewer variables than the dimension $n$. Then

$f \in B^{p} \quad$ if and only if $\sup _{k}\left|a_{k}\right|\left\|P_{n_{k}}\right\|_{\infty}<\infty$. 


\section{MaIn Theorem}

In order to state our main result, we require the so-called Ryll-Wajtaszczyk polynomials. The sequence of such polynomials that we shall need here is slightly different from the original one [7] and was obtained by Ullrich [10, Corollary 1]. We state the existence of those polynomials as a lemma.

Lemma 5. For each $p>0$, there exist a constant $C(p, n)>0$, depending only on $p$ and $n$, and a sequence $\left(W_{k}\right)_{k=1}^{\infty}$ of polynomials in $C^{n}$ homogenous of degree $k$ such that

(i) $\left\|W_{k}\right\|_{\infty} \leqslant 1$, and

(ii) $\left\|W_{k}\right\|_{p} \geqslant C(p, n)$

for every $k=1,2, \cdots$.

We are now ready to state and prove the main result of this paper

MAIN THEOREM. Let $0<p<\infty$ and let $f(z)=\sum_{k=1}^{\infty} a_{k} W_{n_{k}}(z)$ be a holomorphic function on $\mathbb{B}$ with Hadamard gaps (in which each $W_{n_{k}}$ is a Ryll-Wojtaszczyk polynomial that satisfies the two conditions (i) and (ii) of Lemma 5). Then the following conditions are equivalent.

(a) $f \in X^{p}$,

(b) $f \in B^{p}$,

(c) $f \in B_{0}^{p}$,

(d) $\sum_{k=1}^{\infty}\left|a_{k}\right|^{p}<\infty$.

Proof: Since the implications $(c) \Longrightarrow(b) \Longrightarrow(a)$ are trivial, we have only to verify that $(d) \Longrightarrow(c)$ and $(a) \Longrightarrow(d)$. We first assume (d) holds. Then by Lemma 5 (i), we see that

$$
\sum_{k=1}^{\infty}\left|a_{k}\right|^{p}\left\|W_{n_{k}}\right\|_{\infty}^{p} \leqslant \sum_{k=1}^{\infty}\left|a_{k}\right|^{p}
$$

and thus $f \in B_{0}^{p}$ by Lemma 3, which shows the implication $(d) \Longrightarrow(c)$. Finally we assume (a) holds and show (d). From Lemma 2 and Lemma 5 (ii), we get

$$
\infty>\sum_{k=1}^{\infty}\left|a_{k}\right|\left\|W_{n_{k}}\right\|_{p}^{p} \geqslant C(p, n)^{p} \sum_{k=1}^{\infty}\left|a_{k}\right|^{p}
$$

which proves $(a) \Longrightarrow(d)$, and the proof is complete.

The special interest of the Main Theorem is the case $p=2$. So we record it as a corollary. 
COROLLARY. If $f(z)=\sum_{k=1}^{\infty} a_{k} W_{n_{k}}(z)$ is a holomorphic function on $\mathbb{B}$ with Hadamard gaps (in which each $W_{n_{k}}$ is a Ryll-Wojtaszczyk polynomial), then

$$
f \in H^{2} \Longleftrightarrow f \in B M O A \Longleftrightarrow f \in V M O A \Longleftrightarrow \sum_{k=1}^{\infty}\left|a_{k}\right|^{2}<\infty
$$

\section{REFERENCES}

[1] J.S. Choa, 'Some properies of analytic functions on the unit ball with Hadamard gaps', Complex Variables Theory Appl. (to appear).

[2] J.B. Garnett, Bounded analytic functions (Academic Press, New York, 1981).

[3] D.J. Hallenbeck and K. Samotij, 'On radial variation of bounded analytic funcions', Complex Variables Theory Appl. 15 (1990), 43-52.

[4] M. Jevtić, 'On the Carleson measure characterization of BMOA functions on the unit ball', Proc. Amer. Math. Soc. 114 (1992), 379-386.

[5] M. Mateljević and M. Pavlović, ' $L$ - -behavior of power series with positive coefficients and Hardy spaces', Proc. Amer. Math. Soc. 87 (1983), 309-316.

[6] J. Miao, 'A property of analytic functions with Hadamard gaps', Bull. Austral. Math. Soc. 45 (1992), 105-112.

[7] J. Ryll and P. Wojtaszczyk, 'On homogeneous polynomials on a complex ball', Trans. Amer. Math. Soc. 276 (1983), 107-116.

[8] W. Rudin, Function theory in the unit ball of $C^{n}$ (Springer-Verlag, Berlin, Heidelberg, New York, 1980).

[9] C.S. Stanton, 'Counting functions and majorization for Jensen measures', Pacific J. Math. 125 (1986), 459-468.

[10] D. Ullrich, 'Radial divergence in BMOA', Proc. London Math. Soc. 68 (1994), 145-160.

\footnotetext{
Department of Mathematics Education

Sung Kyun Kwan University

Jongro-Gu, Seoul 110-745

Korea

e-mail: jschoa@yurim.skku.ac.kr
} 